\title{
An experimental evaluation of the Agility MLC for motion-compensated VMAT delivery
}

\author{
G A Davies, P Clowes, J L Bedford, P M Evans, S Webb and \\ G Poludniowski \\ Joint Department of Physics, The Institute of Cancer Research and The Royal \\ Marsden NHS Foundation Trust, Downs Road, Sutton, Surrey, SM2 5PT \\ E-mail: gemma.davies@icr.ac.uk
}

\begin{abstract}
An algorithm for dynamic multileaf-collimator (dMLC) tracking of a target performing a known a priori, rigid-body motion during volumetric modulated arc therapy (VMAT), has been experimentally validated and applied to investigate the potential of the Agility (Elekta AB, Stockholm, Sweden) multileaf-collimator for use in motion-compensated VMAT delivery. For five VMAT patients, dosimetric measurements were performed using the Delta ${ }^{4}$ radiation detector (ScandiDos, Uppsala, Sweden) and the accuracy of dMLC tracking was evaluated using a gammaanalysis, with threshold levels of $3 \%$ for dose and $3 \mathrm{~mm}$ for distance-to-agreement. For a motion trajectory with components in two orthogonal directions, the mean gammaanalysis pass rate without tracking was found to be $58.0 \%, 59.0 \%$ and $60.9 \%$ and was increased to $89.1 \%, 88.3 \%$ and $93.1 \%$ with MLC tracking, for time periods of motion of $4 \mathrm{~s}, 6 \mathrm{~s}$ and $10 \mathrm{~s}$ respectively. Simulations were performed to compare the efficiency of the Agility multileaf-collimator with the MLCi multileaf-collimator when used for motion-compensated VMAT delivery for the same treatment plans and motion trajectories. Delivery time increases from a static-tumour to dMLC tracking VMAT delivery were observed in the range $0 \%-20 \%$ for the Agility, and $0 \%-57 \%$ with the MLCi, indicating that the increased leaf speed of the Agility MLC is beneficial for MLC tracking during lung radiotherapy.
\end{abstract}

\section{Introduction}

Dynamic multileaf-collimator (dMLC) tracking as a research interest has led to the development of prototype real-time MLC tracking systems for both Siemens (Tacke et al. 2010, Krauss et al. 2011, Krauss et al. 2012) and Varian (Sawant et al. 2008, Keall et al. 2011, Poulsen et al. 2012a) linear accelerators. It is currently not possible to use Elekta linear accelerators for real-time dMLC tracking, however the feasibility of conformal dMLC tracking of a target performing a known a priori motion trajectory has previously been investigated by McQuaid et al. (2009). In the absence of a realtime MLC tracking system for Elekta linear accelerators, an algorithm for tracking motion known a priori has previously been presented (Davies et al. 2011). In the study presented in this paper, the algorithm is applied to and experimentally validated 
for the latest Elekta multileaf-collimator, Agility (Elekta AB, Stockholm, Sweden), for MLC tracking during volumetric modulated arc therapy (VMAT) (Otto 2008, Yu \& Tang 2011). Few studies so far have investigated the capabilities of the Agility MLC (Cosgrove et al. 2009, Bedford et al. 2013) and the authors believe a study of its suitability for MLC tracking is timely.

The Agility MLC has 160 leaves, of projected leaf width $5 \mathrm{~mm}$ at the isocentre, arranged in two banks of 80 leaves. Each bank of leaves is contained within a dynamic leaf guide (DLG), which can move with the MLC leaves. The maximum velocity of the individual MLC leaves is $35 \mathrm{~mm} \mathrm{~s}^{-1}$, and for the dynamic leaf guide, $30 \mathrm{~mm} \mathrm{~s}^{-1}$, therefore the maximum possible velocity, if both the dynamic leaf guide and the MLC leaves are moving in the same direction, is $65 \mathrm{~mm} \mathrm{~s}^{-1}$. There are two jaws that move perpendicular to the direction of MLC leaf travel, and these have a maximum velocity of $90 \mathrm{~mm} \mathrm{~s}^{-1}$. These increased leaf and jaw velocities compared with previous Elekta multileaf-collimator models, such as the MLCi with a maximum leaf speed of $20 \mathrm{~mm} \mathrm{~s}^{-1}$, could confer advantage for dMLC tracking during VMAT delivery, as is investigated in this paper.

The aim of this study was to experimentally validate the algorithm presented by Davies et al. (2011) for its ability to successfully predict the machine parameters during static-tumour and MLC-tracking VMAT delivery with the Agility MLC, and then to apply this algorithm to investigate the potential of the Agility MLC for motion-compensated VMAT delivery. Dose measurements were performed to determine the dosimetric accuracy of this MLC-tracking technique using the Delta ${ }^{4}$ diode-array phantom, which has previously been evaluated for IMRT and VMAT verification by Bedford et al. (2009). A motion-platform purpose-built in-house for translating the Delta $^{4}$ phantom in three-dimensions was used to simulate target motion. Additionally, through simulations, the delivery-time efficiency for tracking with the Agility multileafcollimator compared with the previous generation multileaf-collimator, the MLCi, was evaluated for the same VMAT plans and motion trajectories.

\section{Method}

\subsection{VMAT treatment plans}

VMAT treatment plans for five lung cancer patients were prepared for the Agility MLC using AutoBeam v5.2, an in-house treatment planning system (Bedford 2009, Bedford 2013). The plans generated were conformal arcs, i.e. the MLC leaves conform to the planning target volume but the prescribed monitor units per segment (i.e. the interval between two neighbouring control points) vary. A case study of the VMAT planning process using AutoBeam has previously been presented (Bedford et al. 2008).

Each plan had a control point spacing of $5^{\circ}$, with a $350^{\circ}$ arc ranging from $185^{\circ}$ to $175^{\circ}$ degrees. The collimator angle for each plan was $0^{\circ}$. When implemented clinically, a collimator of angle of $2^{\circ}-5^{\circ}$ degrees would be used, but this was removed for simplicity 
when transforming the MLC leaf positions to account for motion. A summary of the key parameters for each VMAT plan is given in table 1. In order to provide information regarding the complexity of each treatment plan, the minimum and maximum values of gantry speed, dose rate and prescribed monitor units per segment is presented. The variation in gantry speed and dose rate was measured during delivery of each treatment plan on the linear accelerator used for the experiments presented in this study. It should be noted that the nominal maximum gantry speed of the linear accelerator is $6.0^{\circ} \mathrm{s}^{-1}$, however the transient gantry speed sometimes exceeds this, as is observed in table 1.

Table 1. Key parameters of the VMAT treatment plans used in this study. Values presented are for static-tumour, i.e. without motion-compensation, VMAT delivery.

\begin{tabular}{llllll}
\hline & & & \multicolumn{3}{c}{ Range (min - max) } \\
\cline { 4 - 6 } $\begin{array}{l}\text { Patient } \\
\text { number }\end{array}$ & $\begin{array}{l}\text { Prescribed } \\
\text { dose (Gy) }\end{array}$ & $\begin{array}{l}\text { Monitor } \\
\text { units (MU) }\end{array}$ & $\begin{array}{l}\text { MU per } \\
\text { segment }\end{array}$ & $\begin{array}{l}\text { Gantry speed }^{\dagger} \\
\left({ }^{\circ} \mathrm{s}^{-1}\right)\end{array}$ & $\begin{array}{l}\text { Dose rate }^{\dagger} \\
\left(\text { MU s }^{-1}\right)\end{array}$ \\
\hline 1 & 2.75 & 348.0 & $4.8-5.2$ & $5.7-6.5$ & $5.45-5.98$ \\
2 & 2.75 & 374.9 & $4.5-6.2$ & $5.1-6.4$ & $5.17-6.90$ \\
3 & 2.75 & 408.1 & $5.2-6.4$ & $5.2-6.5$ & $5.95-7.42$ \\
4 & 1.75 & 249.1 & $2.5-4.9$ & $5.3-6.5$ & $2.87-5.62$ \\
5 & 2.75 & 363.1 & $1.6-9.1$ & $5.1-6.8$ & $1.78-9.13$ \\
\hline
\end{tabular}

$\dagger$ Measured data during delivery on linear accelerator.

\subsection{Simulation of a respiratory motion trajectory}

A two-dimensional Lujan et al. (2003) respiratory-motion trajectory was modelled of the form:

$$
\begin{aligned}
& z_{\text {target }}(t)=z_{0}-A_{z} \cos ^{6}\left(\frac{\pi t}{\tau}+\frac{\pi}{2}\right) \\
& y_{\text {target }}(t)=y_{0}+A_{y} \cos ^{6}\left(\frac{\pi t}{\tau}+\frac{3 \pi}{5}\right)
\end{aligned}
$$

where $z_{\text {target }}(t)$ is the motion along the z-axis and $y_{\text {target }}(t)$ is the motion along the y-axis. $z_{0}$ and $y_{0}$ are the rest positions of the target motion (taken to be zero in this study), $A_{z}$ is the amplitude of motion in the z direction and is equal to $20 \mathrm{~mm}$ and $A_{y}$ is the amplitude of motion in the y direction and is equal to $10 \mathrm{~mm}$. The time period is given by $\tau$, and was varied to be either $10 \mathrm{~s}, 6 \mathrm{~s}$ or $4 \mathrm{~s}$ in this set of experiments. A motion platform designed and built in-house for the translation of the Delta ${ }^{4}$ phantom in three-dimensions, and accurate to within $0.2 \mathrm{~mm}$ using a Galil DMC-4060 (Galil Motion Control, Inc., Rocklin, CA, USA) motion controller, was programmed to move with the trajectory described by (1) and (2). The experimental set-up and the orientation of the axes of motion with respect to the linear accelerator are shown in figure 1. 


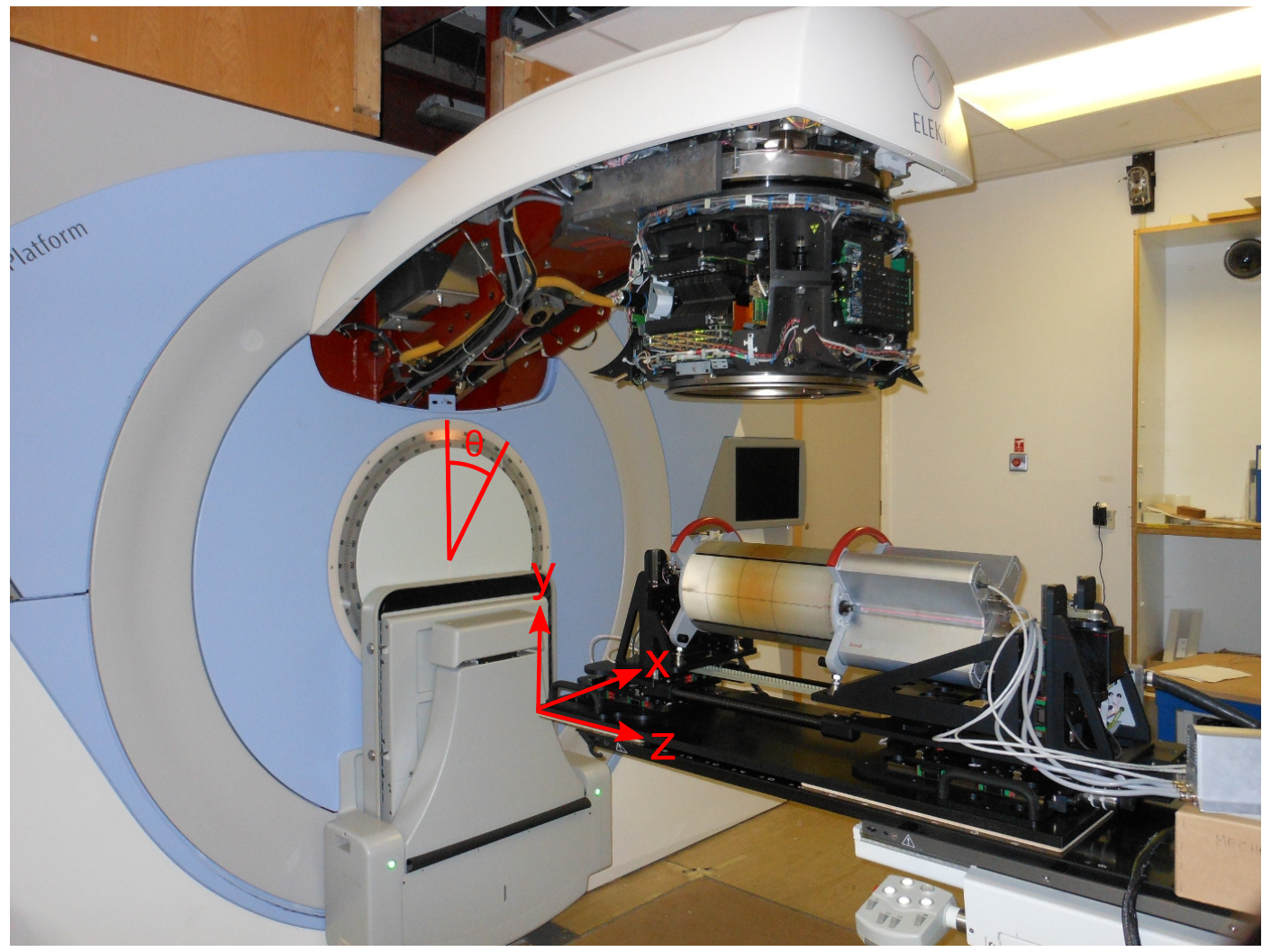

Figure 1. Photograph of the experimental set-up: the motion platform carrying the Delta $^{4}$ is shown mounted on the linear accelerator couch. The orientation of the axes of motion are labelled in red.

\subsection{MLC tracking algorithms}

A VMAT arc is defined as a series of $N$ control points $(n=1,2,3, \ldots, N)$, and a segment $m$ is defined as the interval between two neighbouring control points, $n$ and $n+1$. The total number of segments $(m=1,2,3, \ldots, M)$ is given by $N-1$. The algorithm presented by Davies et al. (2011) compensates for a priori known motion by transforming the planned MLC leaf positions at each control point, and iteratively reducing the dose rate of each segment of the VMAT arc until the MLC tracking plan is deliverable i.e. machine constraints such as maximum leaf velocity and maximum gantry velocity are not violated. In this study, the algorithm was applied to the Integrity 3.0 control system, in which the dose rate can vary by 256 levels, from 0 to the maximum. For motion parallel to the direction of leaf travel the positions of the $k$ MLC leaves, at control point $n, z_{k_{n}}$, were transformed using:

$$
z_{k_{n}}^{\prime}=z_{k_{n}}+f_{\|_{n}}
$$

where for a collimator angle of $0^{\circ}$, the correction required, $f_{\|_{n}}$ is given by: 


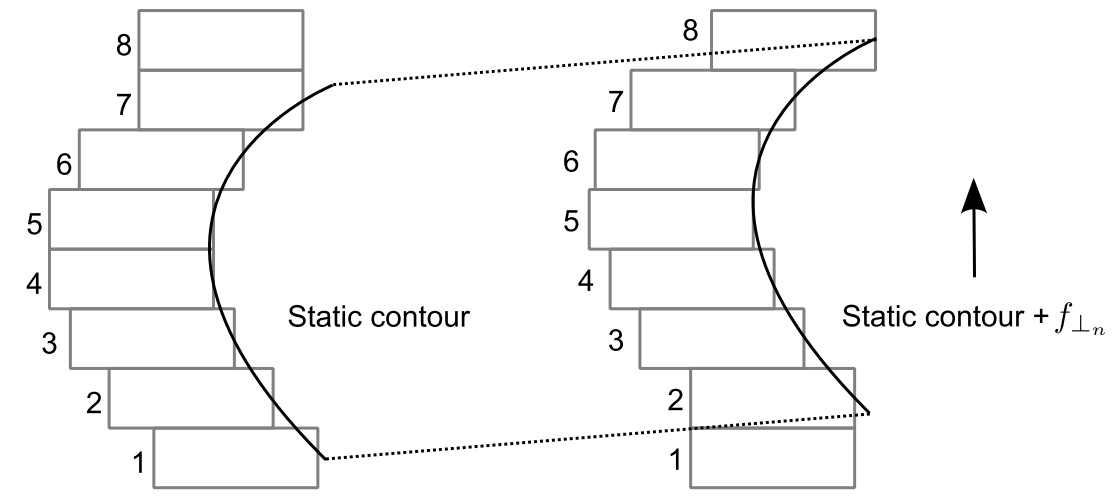

Figure 2. Schematic illustrating the linear-interpolation technique for transforming the MLC aperture to account for motion perpendicular to the direction of leaf travel.

$$
f_{\|_{n}}=y_{\text {target }}\left(t=\sum_{m=1}^{n-1} t_{m}\right) \sin \theta
$$

where $t_{m}$ is the segment time and $\theta$ is the gantry angle, which varies from $0^{\circ}$ to $359^{\circ}$.

For motion perpendicular to the direction of leaf travel, which was not considered in the initial study by Davies et al. (2011), the MLC leaf positions were transformed using a linear-interpolation technique. This involved refitting the MLC leaves to a new contour which was derived from transforming the static MLC aperture contour to account for motion perpendicular to the direction of leaf travel. In this study, the centre of each MLC leaf edge was fitted to its intercept with the new, transformed aperture contour; this is illustrated in figure 2 . The contour shift at control point $n$, perpendicular to the direction of leaf travel, $f_{\perp_{n}}$, is given by:

$$
f_{\perp_{n}}=z_{\text {target }}\left(t=\sum_{m=1}^{n-1} t_{m}\right)
$$

The jaws (denoted Y1 and Y2) that move perpendicular to the direction of leaf travel were also transformed to account for motion using:

$$
Y_{j a w_{n}}^{\prime}=Y_{j a w_{n}}+f_{\perp_{n}}
$$

An example of the aperture transformation, parallel and perpendicular to the direction of leaf travel, at a particular control point for patient 1 is shown in figure 3 . It should be noted that in this study, the direction of motion of the MLC leaves was perpendicular to the motion axis with the largest amplitude, as given by (1). Clinical VMAT plans at our centre are planned in this way, with a collimator rotation close to $0^{\circ}$. This has the advantage of maximising the number of leaves used to create the treatment 
aperture as the apertures are typically elongated in the z-direction and therefore the distance between adjacent MLC leaves at each control point is minimised.

\subsection{Experimental validation of the MLC tracking algorithm with the Agility $M L C$}

The algorithm requires knowledge of certain machine parameters to calculate the statictumour VMAT and dMLC tracking VMAT delivery. These parameters are given in table 2. It should be noted that if all the MLC leaves are moving in the same direction, it is possible for the combined maximum velocity of the MLC leaves and the DLG to be $65 \mathrm{~mm} \mathrm{~s}^{-1}$. However, the main purpose of the dynamic leaf guide is to increase the travel range of the MLC leaves. Therefore, as the VMAT plans used in this study, both static and tracking, required leaf motion between control points typically less than or equal to $20 \mathrm{~mm}$ and of an oscillatory nature, the DLG remained stationary. Therefore the maximum leaf velocity was taken to be $35 \mathrm{~mm} \mathrm{~s}^{-1}$.

Table 2. Machine constraints of the Elekta Synergy linear accelerator used for the measurements.

\begin{tabular}{ll}
\hline Machine parameter & Nominal maximum value \\
\hline Gantry speed $\left({ }^{\circ} \mathrm{s}^{-1}\right)$ & 6 \\
Gantry inertial compensation distance $\left(^{\circ}\right)$ & 5 \\
Dose rate $\left(\mathrm{MU} \mathrm{s}{ }^{-1}\right)$ & 9.25 \\
MLC leaf velocity $\left(\mathrm{mm} \mathrm{s}^{-1}\right)$ & 35 \\
DLG velocity $\left(\mathrm{mm} \mathrm{s}^{-1}\right)$ & 30 \\
Jaw velocity $\left(\mathrm{mm} \mathrm{s}^{-1}\right)$ & 90 \\
\hline
\end{tabular}

To determine the ability of the algorithm to correctly predict the linear accelerator parameters during static-tumour and AMLC VMAT tracking delivery, a logging function on the Elekta linear accelerator control system was used to record the dose rate during each static-tumour and dMLC tracking VMAT delivery. The real-time delivery time, i.e. that measured from the logging function, was compared with the simulated delivery time and the time difference in seconds compared. Additionally, the simulated and real-time dose rates were compared as a function of monitor units for each plan.

\subsection{Evaluation of the accuracy of $d M L C$ tracking}

For each VMAT plan six dose distributions were measured with the Delta ${ }^{4}$ phantom:

(i) Static-tumour VMAT plan with static Delta ${ }^{4}$

(ii) Static-tumour VMAT plan with motion platform translating Delta ${ }^{4}$ in 1D

(iii) Static-tumour VMAT plan with motion platform translating Delta ${ }^{4}$ in $2 \mathrm{D}$

(iv) 1D MLC tracking VMAT plan with motion platform translating Delta ${ }^{4}$ in 1D

(v) 1D MLC tracking VMAT plan with motion platform translating Delta ${ }^{4}$ in 2D

(vi) 2D MLC tracking VMAT plan with motion platform translating Delta ${ }^{4}$ in $2 \mathrm{D}$ 


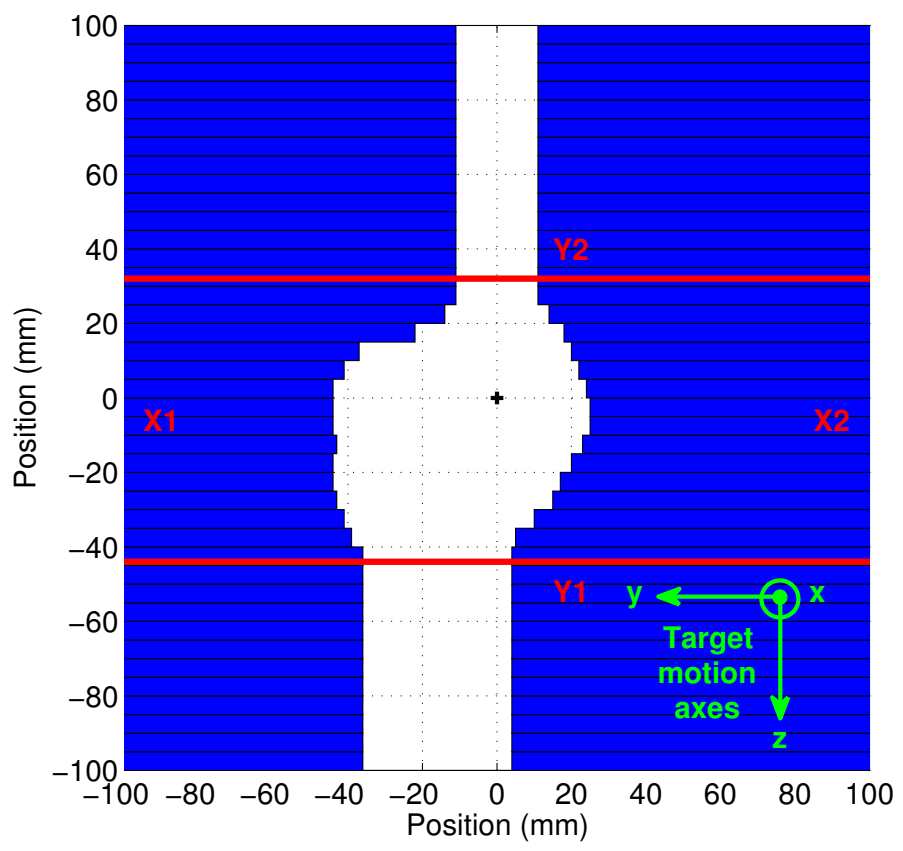

(a)

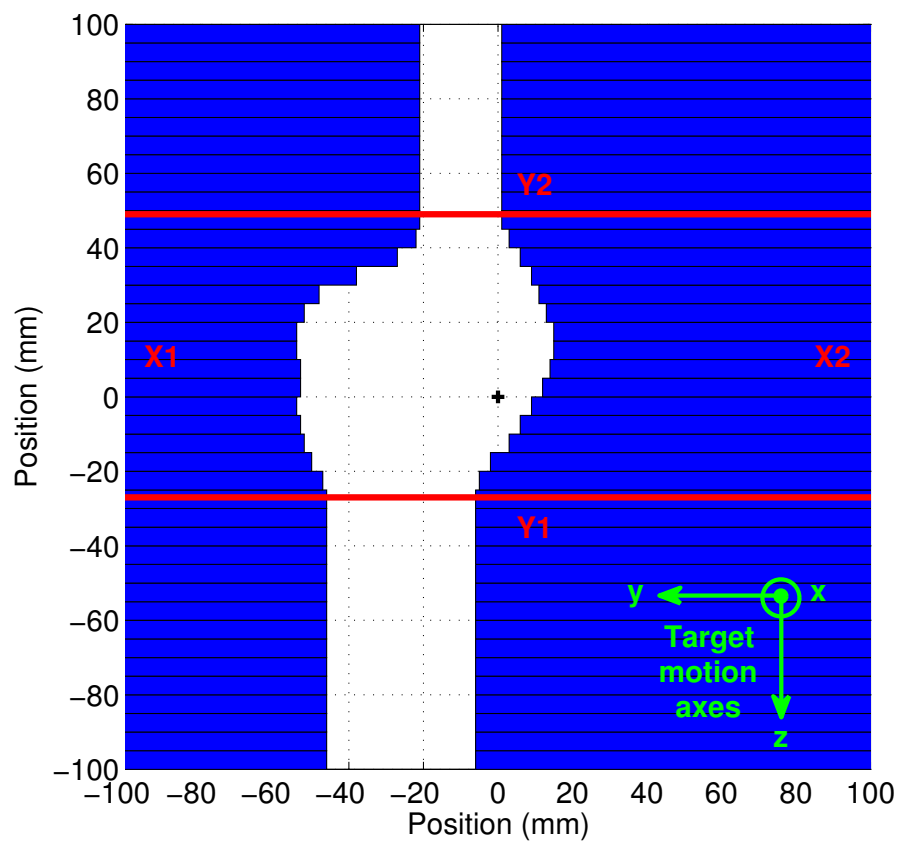

(b)

Figure 3. A diagram showing the beam's-eye-view of the MLC aperture, at a gantry angle of $90^{\circ}$, for patient 1 . The MLC leaves are shown in blue, and the position of the jaws that move perpendicular to the direction of leaf travel, Y1 and Y2, are shown in red. (a) shows the MLC aperture for the static-tumour VMAT plan and (b) shows the MLC aperture for the 2D MLC tracking VMAT plan with $10 \mathrm{~s}$ time period, where the aperture has been transformed for motion along the z-axis and the y-axis. The axes of target motion are labelled in green. The Agility MLC has a total area of $400 \mathrm{~mm}$ $\times 400 \mathrm{~mm}$, but only the inner $200 \mathrm{~mm} \times 200 \mathrm{~mm}$ area is shown here. 
where static-tumour VMAT plans are those created by the treatment planning system, 1D MLC tracking VMAT plans are those where the MLC leaf positions have been adjusted to follow the primary axis of motion in this study (given by (1)), and 2D MLC tracking VMAT plans refer to plans following motion along both axes of motion (given by (1) and (2)).

Dose distributions (ii) and (iv) were acquired with the motion platform translating the Delta ${ }^{4}$ with the motion trajectory given by (1) and dose distributions (iii), (v) and (vi) were acquired with the motion platform translating the Delta ${ }^{4}$ with the motion trajectory given by both (1) and (2). Dose distributions (ii) - (vi) were compared to the reference dose distribution (i) by means of a gamma analysis with a threshold of $3 \%$ and $3 \mathrm{~mm}$. Detectors that were outside of the $5 \%$ isodose level for the reference dose distribution (dose distribution (i)) were not included in the gamma analysis. This was repeated for each of the three time periods of motion investigated in this study. For reference, the dose distribution calculated by the treatment planning system (TPS) for the Delta ${ }^{4}$ geometry was also compared with dose distribution (i) and evaluated using a gamma analysis with threshold levels of $3 \%$ and $3 \mathrm{~mm}$, excluding detectors outside of the $20 \%$ isodose level, as per clinical practice.

There is a finite start-up time between the user initiating the VMAT arc on the control system, and the radiation beam. Therefore, in order to synchronise the movement of the Delta ${ }^{4}$ phantom with gantry movement and thus MLC leaf positions, an optical gating interface was used. The start-up time was determined to be $1.5 \mathrm{~s}$, and therefore as the user initiated the VMAT arc, the motion of the Delta ${ }^{4}$ was triggered, but delayed by $1.5 \mathrm{~s}$ to remove this latency.

\subsection{Evaluation of the delivery efficiency of $M L C$ tracking with the Agility and $M L C i$ multileaf-collimators}

Simulations were performed to compare the increase in delivery time from a statictumour VMAT delivery to a 1D and 2D MLC tracking delivery for the Agility MLC and the MLCi MLC. The MLCi has a lower maximum leaf velocity of $20 \mathrm{~mm} \mathrm{~s}^{-1}$, and a lower maximum jaw velocity. The MLCi jaws that move parallel to the direction of leaf travel have a maximum velocity of $20 \mathrm{~mm} \mathrm{~s}^{-1}$ and those that move perpendicular have a maximum velocity of $15 \mathrm{~mm} \mathrm{~s}^{-1}$. Another major difference is that the MLCi has a leaf width at isocentre of $10 \mathrm{~mm}$, double that of the Agility. However, it was not possible to repeat the experiments performed with the Agility to determine the dosimetric effect of this increased leaf width, as a linear accelerator with an MLCi multileaf-collimator and a version of the Integrity control system was not available at our centre. 


\section{Results}

\subsection{Experimental validation of the $M L C$ tracking algorithm with the Agility $M L C$}

The simulated versus real-time dose rate as a function of monitor units for two of the patients is shown in figure 4. The static-tumour VMAT deliveries are shown in figures 4(a) and 4(c), and the corresponding 2D MLC tracking deliveries, for a time period of $4 \mathrm{~s}$, are shown in figures 4(b) and 4(d). It can be seen that in general, the algorithm correctly predicts the dose rate bin for each segment of the VMAT plan, and the same was observed for all plans measured. The simulated versus real-time delivery time for all plans measured in this set of experiments is shown in figure 5 . The agreement with the line of equality as given by Lin's concordance correlation coefficient (Lin 1989) was found to be 0.914 , indicating strong correlation between the algorithm's predicted value and the measured value. However, it can be seen that the algorithm always underestimates the real-time delivery time. This is due to the fact that the algorithm assumes that the required dose rate bin is reached instantaneously and that the dose rate remains constant at this value for the duration of the segment without fluctuations, which in practice is not the case. Additionally, unscheduled pauses in the treatment beam during the VMAT arc introduce residual time differences between the simulated and the real-time delivery. Such pauses can be seen in figures 4(a) and 4(c), at around $10 \mathrm{MU}$, where the dose rate drops to zero. In the absence of a real-time control system this leads to a degree of desynchronisation between actual motion and the resulting motion-compensation if this occurs during the MLC tracking VMAT deliveries.

\subsection{Evaluation of the accuracy of $M L C$ tracking}

The results of the gamma analysis with threshold levels of $3 \%$ for dose and $3 \mathrm{~mm}$ for distance-to-agreement are shown in figure 6 . Additionally, the results averaged over the five patient plans investigated in this study are presented in table 3. Figure 6 shows that for all patient plans, the use of MLC tracking, either 1D or 2D, has improved the gammaanalysis pass rate compared to the pass rate without tracking, and the improvement is most pronounced for the $10 \mathrm{~s}$ time period motion trajectory. This result is expected, as the differences in the simulated and real-time delivery due to the limitations of the model (discussed in the previous section) will be less important for the longer time period. For example, a $1 \mathrm{~s}$ time difference corresponds to a tenth of a breathing cycle for the $10 \mathrm{~s}$ tracking plans but will correspond to a quarter of a breathing cycle for the $4 \mathrm{~s}$ tracking plans. The results of the gamma-analysis reflect this; the mean pass rate for 1D tracking with $1 \mathrm{D}$ motion and $2 \mathrm{D}$ tracking with $2 \mathrm{D}$ motion is $93.0 \%$ and $93.1 \%$ respectively for a $10 \mathrm{~s}$ time period, and is reduced to $84.2 \%$ and $89.1 \%$ for a $4 \mathrm{~s}$ time period. Additionally, it was found that the gamma analysis pass rate for the dose distribution calculated by the TPS for the Delta ${ }^{4}$ compared with the measured static dose distribution was found to be $100 \%$ for all patients.

In general, the highest gamma-analysis pass rate is seen for the $1 \mathrm{D}$ tracking with 


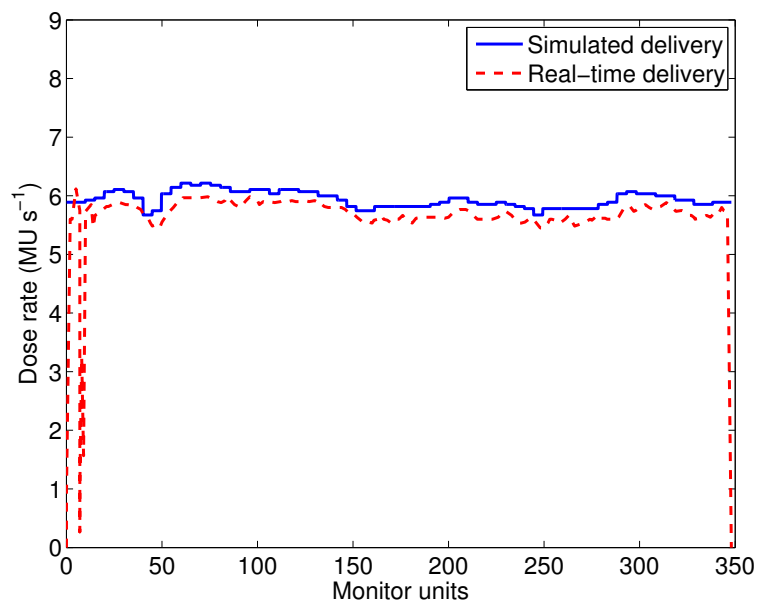

(a)

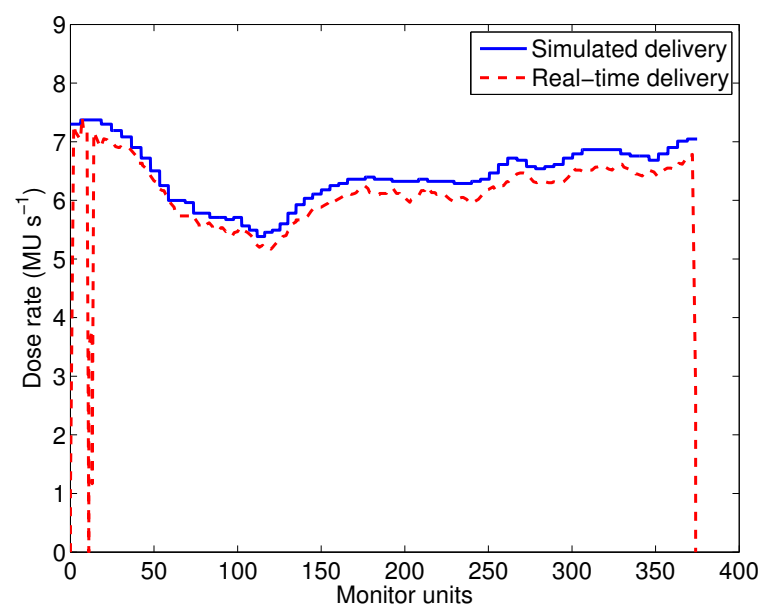

(c)

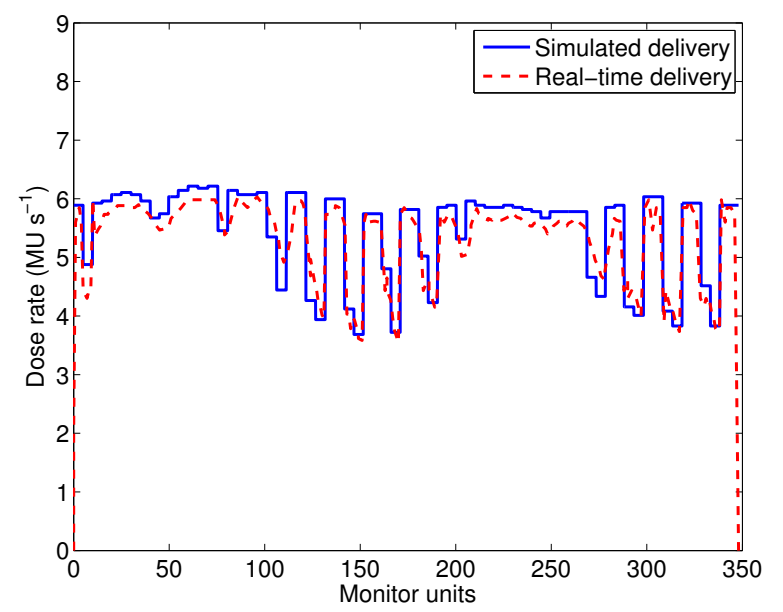

(b)

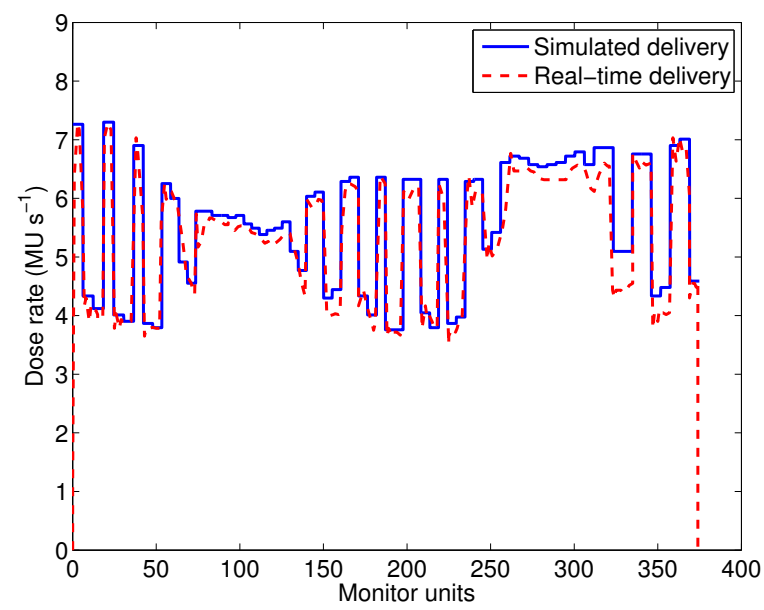

(d)

Figure 4. Dose rate as a function of monitor units for simulated and real-time VMAT delivery. The dose rate for the static-tumour VMAT plans for patient 1 and patient 2 are shown in (a) and (c) respectively, and the corresponding 2D MLC tracking VMAT plans (time period of $4 \mathrm{~s}$ ) are shown in (b) and (d).

1D motion delivery and 2D tracking with 2D motion delivery, with some exceptions. During the experiments, if an unscheduled pause occurred in the VMAT arc, no attempt was made to repeat the arc until it delivered without pauses. The VMAT arc was only repeated if a fault brought the treatment to a premature end. Therefore any unscheduled pauses in the radiation beam resulted in the desynchronisation of the MLC leaf positions and the motion of the Delta ${ }^{4}$, and the acquired dose distribution still displayed minor motion-blurring. Therefore the gamma-analysis pass rate for certain tracking plans was reduced. For example, for patient 1 for a time period of $6 \mathrm{~s}$, the 1D tracking with 1D motion delivery had the lowest gamma-analysis pass rate of all the tracking deliveries. The time difference between real-time and simulated delivery was $1.9 \mathrm{~s}$ for 1D tracking with $1 \mathrm{D}$ motion, $0.9 \mathrm{~s}$ for $2 \mathrm{D}$ motion with $1 \mathrm{D}$ tracking and $0.6 \mathrm{~s}$ for the $2 \mathrm{D}$ tracking 


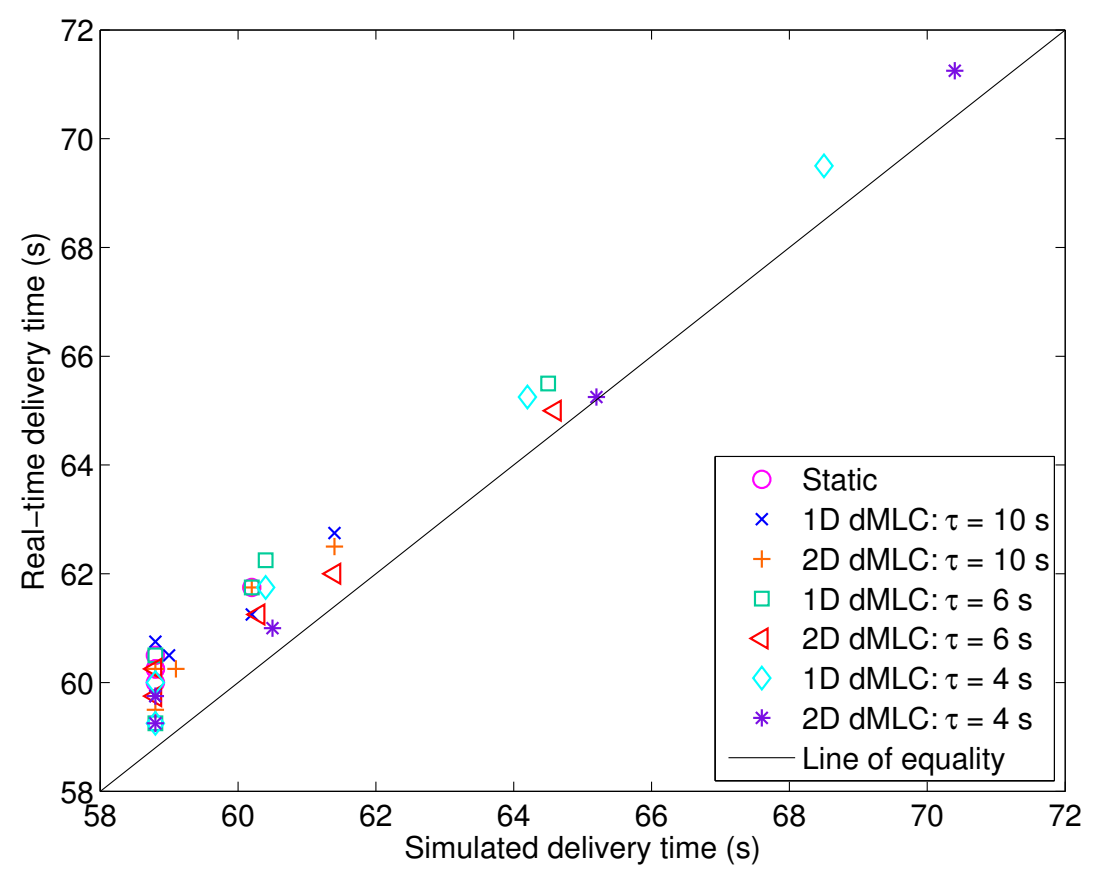

Figure 5. Comparison of the real-time delivery time and the simulated delivery time for both static-tumour and MLC tracking plans for all patients.

with 2D motion delivery. Therefore, the first delivery with the largest time difference received the lowest gamma-analysis pass rate. For patient 5 , for a $6 \mathrm{~s}$ time period, 1D tracking with $2 \mathrm{D}$ motion received the highest pass rate, and the same is observed for patient 2 for a $10 \mathrm{~s}$ time period. For patient 5, the time difference between simulated and real-time was $1.6 \mathrm{~s}$ for $1 \mathrm{D}$ tracking with $1 \mathrm{D}$ motion, $0.6 \mathrm{~s}$ for $1 \mathrm{D}$ tracking with $2 \mathrm{D}$ motion and $1.0 \mathrm{~s}$ for $2 \mathrm{D}$ tracking with $2 \mathrm{D}$ motion and the analogous time differences were $1.4 \mathrm{~s}, 0.6 \mathrm{~s}$ and $1.1 \mathrm{~s}$ for patient 2 . This explains why $1 \mathrm{D}$ tracking with $2 \mathrm{D}$ motion had the highest pass rate for these examples.

Despite these explicable discrepancies, the results show that when the VMAT arc is delivered smoothly without interruptions, the algorithm works well and a pass rate over $90 \%$ can be achieved, but unscheduled pauses are detrimental to the tracking dose distribution. For the sinusoidal motion studied here, with real-time control of the radiation beam, it would be possible to correct for the desynchronisation caused by these unscheduled pauses. For example, the breathing phase of the motion platform, and the corresponding breathing phase of the MLC leaf positions could be continually monitored, and if substantial desynchronisation is observed, the radiation beam could be paused until the correct phase is reached by the motion platform, at which point the beam could be reinitialised. However, such real-time control of the radiation beam was not available for this set of experiments, but would be desirable for further work with this algorithm. 
Table 3. Gamma analysis results averaged over five patient plans. Numbers are percentages, presented as mean \pm standard deviation.

\begin{tabular}{llllll}
\hline & \multicolumn{5}{c}{ Gamma analysis percentage pass rate $(3 \%$ and $3 \mathrm{~mm})$} \\
\cline { 2 - 6 } & 1D motion & 2D motion & 1D motion & 2D motion & 2D motion \\
Time period (s) & No tracking & No tracking & 1D tracking & 1D tracking & 2D tracking \\
\hline 4 & $60.3 \pm 8.6$ & $58.0 \pm 6.9$ & $84.2 \pm 7.5$ & $75.1 \pm 2.8$ & $89.1 \pm 4.3$ \\
6 & $61.5 \pm 7.9$ & $59.0 \pm 7.3$ & $85.4 \pm 9.9$ & $88.3 \pm 3.9$ & $88.3 \pm 7.4$ \\
10 & $62.9 \pm 6.3$ & $60.9 \pm 7.4$ & $93.0 \pm 3.9$ & $89.7 \pm 4.2$ & $93.1 \pm 3.4$ \\
\hline
\end{tabular}

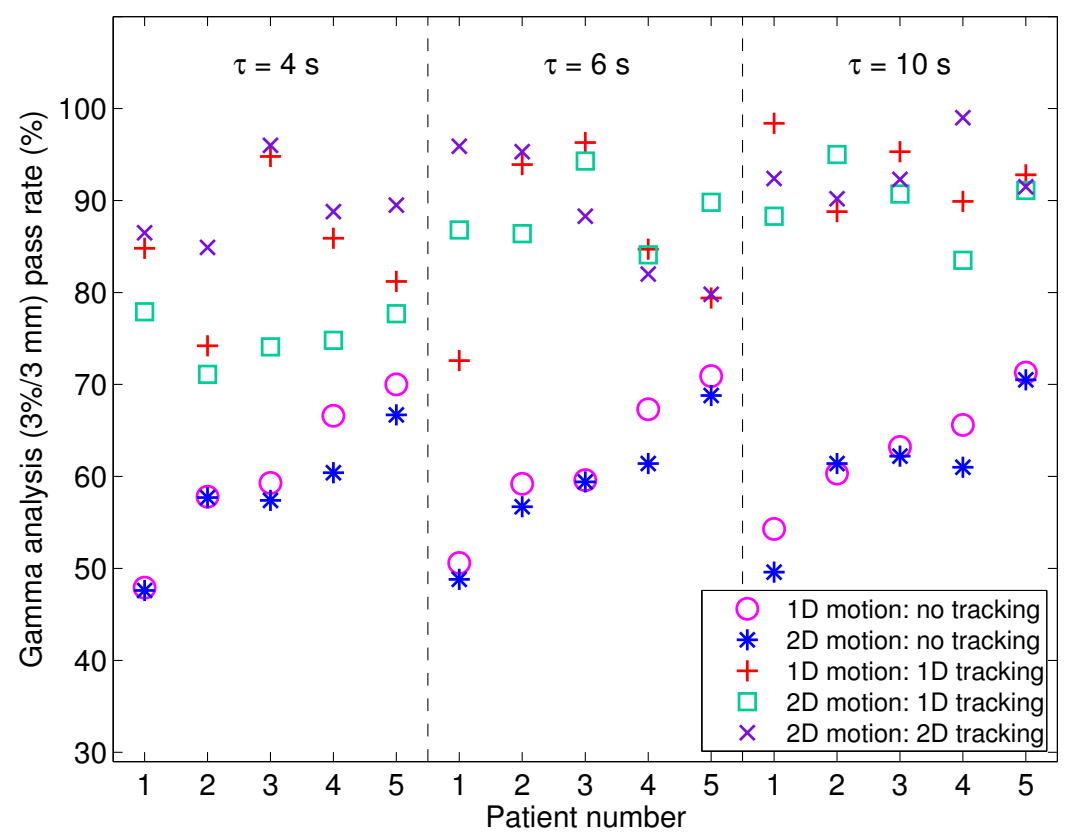

Figure 6. Gamma analysis results from the dosimetric accuracy experiments with the Delta $^{4}$ radiation detector. A threshold level of $3 \%$ for dose and $3 \mathrm{~mm}$ for distanceto-agreement was used, and detectors outside of the $5 \%$ isodose level in the reference dose distribution were not included in the analysis.

\subsection{Evaluation of the delivery efficiency of $M L C$ tracking with the Agility and $M L C i$ multileaf-collimator}

The results of the simulations performed to compare the delivery times of the MLCi multileaf-collimator and Agility multileaf-collimator for the same VMAT plans are shown in figure 7 . It can be seen that the static-tumour VMAT delivery times are comparable for both the MLCi and the Agility MLC, but the Agility MLC is able to deliver the tracking plans in a shorter treatment time. In particular, for patients 3 to 5 , the Agility MLC was able to deliver the tracking plans without an increase in delivery time, which was not possible with the MLCi MLC. For both multileaf-collimators, the largest percentage time increase was observed for the 2D MLC tracking VMAT plans 


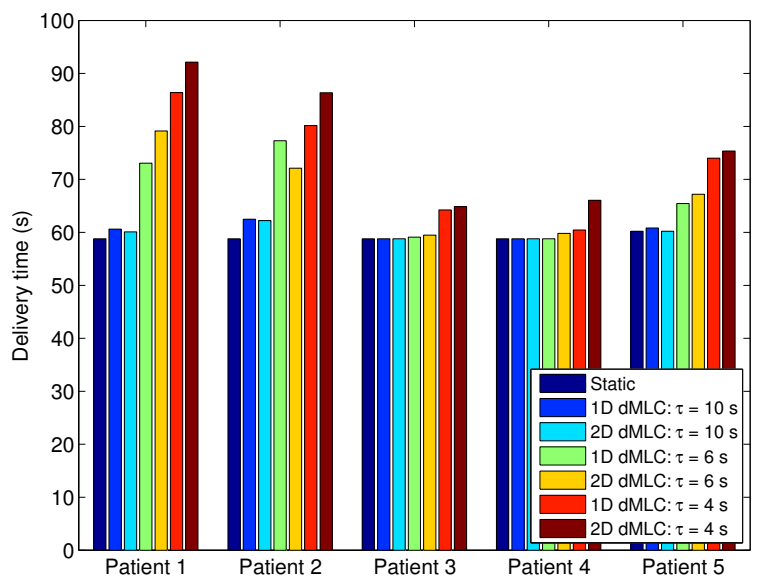

(a)

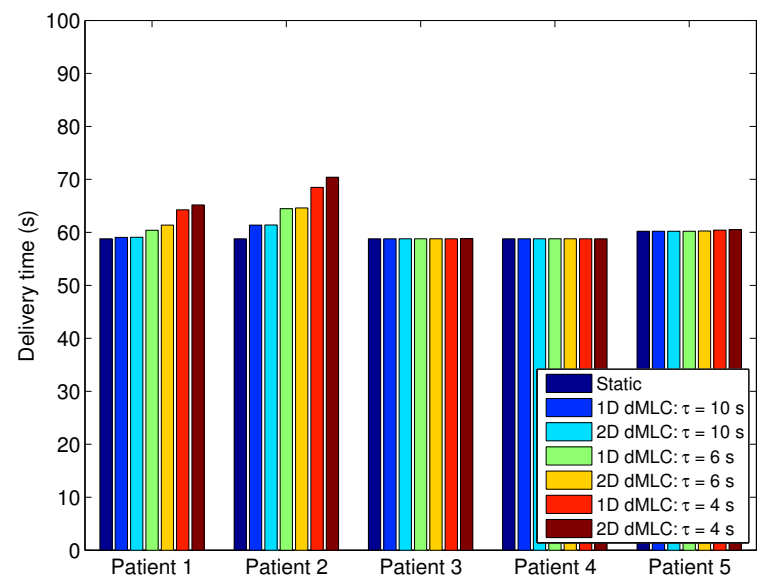

(b)

Figure 7. Simulated absolute delivery times for the static and tracking plans used in this study for (a) the MLCi multileaf-collimator and (b) the Agility multileafcollimator.

for the shortest time period motion trajectory. The greatest percentage time increase observed from a static-tumour to MLC tracking delivery for the MLCi was 57\%, for patient 1 , and the analogous time increase for the Agility was $11 \%$. It is clear that the Agility MLC leaf speed of $35 \mathrm{~mm} \mathrm{~s}^{-1}$ and increased jaw velocity, compared with the MLCi leaf speed of $20 \mathrm{~mm} \mathrm{~s}^{-1}$ and lower jaw velocity, is advantageous for MLC tracking, particularly with decreased time period of motion.

\section{Discussion}

In this study, a theoretical algorithm has been applied, experimentally, to evaluate MLC tracking during VMAT delivery on Elekta linear accelerators for the first time. VMAT MLC tracking experiments have also been performed and presented for Varian linear accelerators (Sawant et al. 2008, Zimmerman et al. 2009, Falk et al. 2010, Keall et al. 2011, Poulsen et al. 2012b). However, these experiments were performed using a real-time MLC tracking system, which is not possible with the Elekta system used here, and limited the success of the results presented in this paper. Keall et al. (2011) performed real-time dMLC IMAT (no dose rate or gantry speed modulation) tracking measurements of realistic lung traces with the Varian MLC tracking system, and found, on average, that $1.6 \%$ of points failed the $3 \% / 3 \mathrm{~mm}$ gamma analysis with tracking. Additionally, they found no loss of treatment efficiency for the two lung plans investigated, but these were for IMAT plans without modulation of gantry speed and dose rate, whereas all plans used in the study presented here featured modulation of the gantry speed and dose rate. Zimmerman et al. (2009) have presented dMLC tracking results for one lung VMAT plan on a Varian accelerator, also using the Delta ${ }^{4}$ detector, and showed an increase in the gamma-analysis pass rate from $87 \%$ without 
tracking to $97 \%$ with tracking, but the efficiency of the treatment was not mentioned. Poulsen et al. (2012b) also demonstrated successful image-based MLC tracking of two lung VMAT plans with dose rate and gantry modulation on a Varian system (mean gamma pass rate of $98.3 \%$ ), but again the efficiency was not mentioned. None of the measurements performed in the study in this paper achieved the same accuracy level for VMAT MLC tracking, although the mean gamma-analysis pass rate with $2 \mathrm{D}$ motioncompensation was on the border of clinical acceptability (Ezzell et al. 2009) for all time periods investigated. One of the contributing factors towards this result, was that it was only possible to transform the MLC leaf positions to follow a target motion at the control points (every $5^{\circ}$ for these plans) and therefore the time interval at which the transformation took place varied with gantry speed. Such coarse and irregular sampling of the breathing cycle left a residual error between the position of the target and the transformed position of the MLC aperture, as between control points, the MLC motion was linear but the target motion, non-linear. It is possible to deliver a VMAT arc on Elekta linear accelerators with a finer control point spacing, e.g. $2^{\circ}$, which has been shown theoretically to improve the accuracy of tracking compared with coarser control points (Davies et al. 2011). However, for this experimental study, plans of control point spacing $5^{\circ}$ were used as the ability of the algorithm to correctly predict delivery parameters during a VMAT arc was shown to increase with increased control point spacing (Davies et al. 2011). The limitations of transforming the leaf positions only at the control points compromised the tracking accuracy the most for the trajectory with the shortest time period, and therefore the highest gamma-analysis pass rates were seen for the motion trajectory with $10 \mathrm{~s}$ time period, where the breathing cycle was sampled most frequently. However, as shown by Seppenwoolde et al. (2002) in an analysis of the characteristics of lung-tumour motion, the average time period is $3.6 \mathrm{~s}$. Therefore, for accurate MLC tracking of realistic respiratory motion it would be desirable to be able to transform the leaf positions at a specified time interval, as seen in prototype real-time MLC tracking systems (Keall et al. 2006, Tacke et al. 2010), rather than be constrained solely to the control points.

The tracking algorithm presented here reduces the dose rate until the MLC tracking plan is deliverable. However, Bedford \& Warrington (2009) have shown that at low dose rates beam symmetry can be degraded, and recommend that dose rates less than 1.25 $\mathrm{MU} \mathrm{s}^{-1}$ should only be used for small fractions of the VMAT delivery. For the plans used in this study, the majority of the deliveries, both static-tumour and MLC tracking, were delivered at a dose rate greater than $3 \mathrm{MU} \mathrm{s}^{-1}$ with the lowest observed dose rate being $1.75 \mathrm{MU} \mathrm{s}^{-1}$ for the plans for patient 5 , for a duration of approximately $10 \mathrm{MU}$. Therefore beam symmetry was not an issue in these experiments, but is something to be cautious of when implementing this algorithm.

The main limitation of the algorithm used in this study, is the requirement for $a$ priori knowledge of the patient's respiratory motion. Respiration can be unpredictable (Seppenwoolde et al. 2002, Shirato et al. 2006), and therefore there could be difficulty in translating this algorithm to a clinical setting, where the motion may vary from that 
seen during the radiotherapy planning process. It is possible to account for changes in breathing frequency using adaptive dose-rate regulation as demonstrated by $\mathrm{Yi}$ et al. (2008), which could be used to maintain synchronisation between the planned MLC motion (prescribed as a function of monitor units) and the tumour motion. However, amplitude variations and baseline shifts are more problematic, and difficult to correct with this method. The ideal solution to such a problem is detection of the motion in real-time and transformation of the MLC leaf positions accordingly, allowing pauses in the radiation beam if the tumour motion is too fast for the MLC leaves to track, as described by Sawant et al. (2008). However, real-time MLC tracking is not trivial; accounting for system latency and reliable detection of tumour position are some of the difficulties faced.

The plans investigated in this study all had gantry speed and dose rate modulation, and the greatest increase in delivery time observed was $20 \%$, and for three of the patients, there was a negligible increase in delivery time. The results therefore demonstrate that the Agility MLC has the potential to be a powerful and improved tool for MLC tracking due to its increased leaf and jaw speeds. Although the Agility MLC design has the additional benefit of the dynamic leaf guide, for this set of experiments and the VMAT plans selected, the DLG did not prove to be useful. Whether this is the case for all VMAT plans, or for other treatment modalities cannot be concluded from the experiments performed, and it is possible that for step-and-shoot IMRT delivery, or conformal delivery, the DLG would be a desirable feature for MLC tracking. It was not possible in this study to evaluate the dosimetric benefit of the thinner leaf width of the Agility compared with the MLCi multileaf collimator, but as shown by Poulsen et al. (2012b), the areas of underdose and overdose are directly correlated with the gamma-analysis pass rate, and therefore one would expect that an MLC with a thinner leaf width would be more suitable for MLC tracking, where it is often necessary to transform the MLC apertures parallel to the direction of leaf travel.

Although the leaf speed of Agility MLC is one of the fastest commercially available, it should be noted that this increased speed enables greater leaf shifts which, if justified, must be modelled correctly in the treatment planning process. As illustrated in a review by Yu \& Tang (2011), plans with longer leaf travel can result in greater discrepancies between the planned and delivered dose, and therefore it is vital that sufficient beam sampling is allowed in the dose calculation to ensure that the treatment planning system calculation is representative of the planned delivery.

\section{Conclusion}

An algorithm for MLC tracking VMAT delivery on Elekta linear accelerators has been experimentally validated for the Agility MLC with the Integrity 3.0 control system, and dosimetric measurements have been performed to evaluate the accuracy of MLC tracking with this technique. It has been shown that an a priori method can offer accurate and efficient motion-compensation, with the majority of MLC tracking deliveries carried 
out with a modest, or zero, time increase compared with the static-tumour delivery. However, a real-time system is necessary for increased accuracy and to take full advantage of the Agility MLC's capabilities.

\section{Acknowledgements}

The authors would like to thank Chris Bunton for collaborating with Peter Clowes on the design and construction of the motion platform and Elekta for useful discussions regarding the behaviour of the Agility MLC. This work was supported by an ICR PhD studentship and Cancer Research UK (grant number C46/A10588).

\section{References}

Bedford J L 2009 Treatment planning for volumetric modulated arc therapy. Med Phys 36(11), 51285138 .

Bedford J L 2013 Sinogram analysis of aperture optimization by iterative least-squares in volumetric modulated arc therapy. Phys Med Biol 58(5), 1235-1250.

Bedford J L, Lee Y K, Wai P, South C P \& Warrington A P 2009 Evaluation of the Delta4 phantom for IMRT and VMAT verification. Phys Med Biol 54(9), N167-76.

Bedford J L, Nordmark Hansen V, McNair H A, Aitken A H, Brock J E C, Warrington A P \& Brada M 2008 Treatment of lung cancer using volumetric modulated arc therapy and image guidance: a case study. Acta Oncol 47(7), 1438-1443.

Bedford J L, Thomas M D R \& Smyth G 2013 Beam modeling and VMAT performance with the Agility 160-leaf multileaf collimator. J Appl Clin Med Phys 14(2), 172-185.

Bedford J L \& Warrington A P 2009 Commissioning of volumetric modulated arc therapy (VMAT). Int $J$ Radiat Oncol Biol Phys 73(2), 537-545.

Cosgrove V, Thomas M, Weston S, Thompson M, Reynaert N, Evans C, K.J. B, De Wagter C, Thwaites D \& Warrington A 2009 Physical Characterization of a New Concept Design of an Elekta Radiation Head with Integrated 160-leaf Multi-leaf Collimator Int J Radiat Oncol Biol Phys 75(3), S722-S723.

Davies G A, Poludniowski G \& Webb S 2011 MLC tracking for Elekta VMAT: a modelling study. Phys Med Biol 56(23), 7541-54.

Ezzell G A, Burmeister J W, Dogan N, LoSasso T J, Mechalakos J G, Mihailidis D, Molineu A, Palta J R, Ramsey C R, Salter B J, Shi J, Xia P, Yue N J \& Xiao Y 2009 IMRT commissioning: multiple institution planning and dosimetry comparisons, a report from AAPM Task Group 119. Med Phys 36(11), 5359-73.

Falk M, af Rosenschöld P M, Keall P, Cattell H, Cho B C, Poulsen P, Povzner S, Sawant A, Zimmerman J \& Korreman S 2010 Real-time dynamic MLC tracking for inversely optimized arc radiotherapy. Radiother Oncol 94(2), 218-23.

Keall P J, Cattell H, Pokhrel D, Dieterich S, Wong K H, Murphy M J, Vedam S S, Wijesooriya K \& Mohan R 2006 Geometric accuracy of a real-time target tracking system with dynamic multileaf collimator tracking system. Int J Radiat Oncol Biol Phys 65(5), 1579-84.

Keall P J, Sawant A, Cho B, Ruan D, Wu J, Poulsen P, Petersen J, Newell L J, Cattell H \& Korreman S 2011 Electromagnetic-Guided Dynamic Multileaf Collimator Tracking Enables Motion Management for Intensity-Modulated Arc Therapy. Int J Radiat Oncol Biol Phys 79(1), 312-320.

Krauss A, Fast M F, Nill S \& Oelfke U 2012 Multileaf collimator tracking integrated with a novel x-ray imaging system and external surrogate monitoring. Phys Med Biol 57(8), 2425-39. 
Krauss A, Nill S, Tacke M \& Oelfke U 2011 Electromagnetic real-time tumor position monitoring and dynamic multileaf collimator tracking using a Siemens 160 MLC: geometric and dosimetric accuracy of an integrated system. Int J Radiat Oncol Biol Phys 79(2), 579-87.

Lin L I 1989 A concordance correlation coefficient to evaluate reproducibility. Biometrics 45(1), 255-68.

Lujan A E, Balter J M \& Ten Haken R K 2003 A method for incorporating organ motion due to breathing into 3D dose calculations in the liver: sensitivity to variations in motion. Med Phys 30(10), 2643-2649.

McQuaid D, Partridge M, Symonds-Tayler J R, Evans P M \& Webb S 2009 Target-tracking deliveries on an Elekta linac: a feasibility study. Phys Med Biol 54(11), 3563-3578.

Otto K 2008 Volumetric modulated arc therapy: IMRT in a single gantry arc. Med Phys 35(1), 310-317. Poulsen P R, Carl J, Nielsen J, Nielsen M S, Thomsen J B, Jensen H K, Kjærgaard B, Zepernick P R, Worm E, Fledelius W, Cho B, Sawant A, Ruan D \& Keall P J $2012 a$ Megavoltage image-based dynamic multileaf collimator tracking of a NiTi stent in porcine lungs on a linear accelerator. Int J Radiat Oncol Biol Phys 82(2), e321-7.

Poulsen P R, Fledelius W, Cho B \& Keall P $2012 b$ Image-based dynamic multileaf collimator tracking of moving targets during intensity-modulated arc therapy. Int J Radiat Oncol Biol Phys 83(2), e265-71.

Sawant A, Venkat R, Srivastava V, Carlson D, Povzner S, Cattell H \& Keall P 2008 Management of three-dimensional intrafraction motion through real-time DMLC tracking. Med Phys 35(5), 2050-2061.

Seppenwoolde Y, Shirato H, Kitamura K, Shimizu S, van Herk M, Lebesque J V \& Miyasaka K 2002 Precise and real-time measurement of 3D tumor motion in lung due to breathing and heartbeat, measured during radiotherapy. Int J Radiat Oncol Biol Phys 53(4), 822-834.

Shirato H, Suzuki K, Sharp G C, Fujita K, Onimaru R, Fujino M, Kato N, Osaka Y, Kinoshita R, Taguchi H, Onodera S \& Miyasaka K 2006 Speed and amplitude of lung tumor motion precisely detected in four-dimensional setup and in real-time tumor-tracking radiotherapy. Int $J$ Radiat Oncol Biol Phys 64(4), 1229-36.

Tacke M B, Nill S, Krauss A \& Oelfke U 2010 Real-time tumor tracking: Automatic compensation of target motion using the Siemens 160 MLC Med Phys 37(2), 753-761.

Yi B Y, Han-Oh S, Lerma F, Berman B L \& Yu C 2008 Real-time tumor tracking with preprogrammed dynamic multileaf-collimator motion and adaptive dose-rate regulation. Med Phys 35(9), 39553962.

Yu C X \& Tang G 2011 Intensity-modulated arc therapy: principles, technologies and clinical implementation. Phys Med Biol 56(5), R31-R54.

Zimmerman J, Korreman S, Persson G, Cattell H, Svatos M, Sawant A, Venkat R, Carlson D \& Keall P 2009 DMLC motion tracking of moving targets for intensity modulated arc therapy treatment: a feasibility study. Acta Oncol 48(2), 245-250. 